\title{
Thymian - Arzneipflanze des Jahres 2006
}

\author{
Felix Iten, Reinhard Saller \\ Institut für Naturheilkunde, UniversitätsSpital Zürich, CH-Zürich
}

D er Thymian (Thymus vulgaris), auch Echter Thymian, Gartenthymian, Gemeiner Thymian oder Römischer Thymian genannt, wurde vom Würzburger Studienkreis „Entwicklungsgeschichte der Arzneipflanzenkunde“ zur Arzneipflanze des Jahres 2006 erkoren. Der Thymian, jedermann bekannt als Küchengewürz, ist als Arzneipflanze - verglichen mit seiner Bekanntheit als Küchengewürz - eine nahezu unbekannte Grösse. Zu Unrecht, wie die Forschung der letzten Jahrzehnte, die sich mit dem Thymian beschäftigte, zeigt. Denn dank dem wachsenden Interesse an therapeutischen Anwendungen ätherischer öle (Aromatherapie), der Suche nach natürlichen Konservierungsstoffen in der Lebensmitteltechnologie und der hochaktuellen Problematik der Antibiotikaresistenz stehen ätherischölhaltige Pflanzen vermehrt im Blickfeld der naturwissenschaftlichen Forschung. Die Forschung hat einige der traditionellen Anwendungen des Thymians bestätigt, aber auch neue pharmakologische Eigenschaften ans Tageslicht gebracht. Mit der Wahl des Thymians trägt der Würzburger Studienkreis diesen neuen Erkenntnissen aus der noch jungen Thymianforschung Rechnung.

\section{Botanik}

Der Echte Thymian (Thymus vulgaris) ist ein reich verzweigter, bis $40 \mathrm{~cm}$ hoher, Zwergstrauch (Abb.1). Die eingerollten Blattränder und die starke Behaarung auf der Unterseite der Blätter sind Anpassungen an das heisse und trockene Klima seines Herkunftgebietes, der mediterranen Strauch-
Der Echte Thymian (Thymus vulgaris) wurde vom Würzburger Studienkreis „Entwicklungsgeschichte der Arzneipflanzenkunde" zur Arzneipflanze des Jahres 2006 erkoren. Was verschafft dem Thymian diese Ehre? Neben der langen Tradition als Arzneipflanze mit Indikationen, die sich zum Teil bis heute gehalten haben (z.B.: Keuchhusten und Bronchitis), förderte die jüngste Forschung eine ganze Reihe neuer viel versprechender Eigenschaften des Thymians zu Tage, die die Wahl zur Arzneipflanze 2006 zweifellos rechtfertigen. Thymol und Carvacrol, die beiden Hauptwirkstoffe des ätherischen Öls, aber auch das ätherische Öl selbst sowie Extrakte des Thymians, zeichnen sich durch eine hohe antimikrobielle Aktivität und ein breites Wirkspektrum aus. Die antiseptischen Eigenschaften des Thymians kommen bei den unterschiedlichsten Anwendungen zum Tragen: bei Bronchitis, zum Gurgeln bei entzündeten Schleimhäuten in Mund- und Rachenbereich, bei infizierten Wunden und äusserlichen Pilzinfektionen aber auch bei der Bekämpfung Akarizid-resistenter Varroa-Milben oder als Konservierungsmittel in der Nahrungsmitteltechnologie. Weitere Eigenschaften, die durch experimentelle Daten gestützt werden, sind die antiphlogistische, die antioxidative, die spasmolytische, die sekretolytische und die tonisiernde Wirkung. Die aktuelle Forschung zeigt, dass das Potential des Vielstoffgemisches Thymian noch lange nicht ausgeschöpft ist. Ein Bereich, der zunehmend von der experimentellen Forschung entdeckt wird, sind Vielstoffgemische und deren Fraktionen, die mitunter Eigenschaften aufweisen, die den isolierten Wirkstoffen fehlen. Auf weitere Ergebnisse der noch jungen Thymianforschung darf man gespannt sein.

Schlüsselwörter: Thymian, Quendel, Geschichte, Vielstoffgemisch, antimikrobielle Aktivität, antimykotisch, antioxidativ, antiphlogistisch, Repellent, akarizid

\section{Thyme - Medical Plant of the Year 2006}

The Würzburg study group „Entwicklungsgeschichte der Arzneipflanzenkunde” (History of the development of medicinal plant botany) nominated Thyme (Thymus vulgaris) as the medical plant of the year 2006. Is this honour justified? The long traditional use and the long history of its most important indications (Whooping Cough and bronchitis) which have been scientifically proven in the meantime, as well as the results of the recent years' research, revealing many new properties of thyme and its ingredients, legitimate this choice by far. Thymole and Carvacrole, the main compounds of the essential oil, as well as the essential oil itself, exhibit strong antimicrobial activity against a broad range of micro-organisms. The antiseptic property of thyme plays an important part in many different applications: early stage of bronchitis, inflamed mucosa of mouth and throat, small infected wounds of the skin and external mycosis. Non-medical applications, for example, its use against acaricide-resistant Varroa mites has already been established and its efficacy as a preservative in food technology is under investigation. Further properties that are well documented by experimental research are: antiinflammatory, antioxydative, spasmolytic, secretolytic and tonifying effects. The current research on thyme indicates that the potential of this multi-compound drug is far from being exhausted. A domain that gains increasing relevance in experimental research is the exploration of multi-component drugs as a whole or of their specific fraction because they might possess properties that the single active components lack. Further interesting and encouraging results in thyme research are anticipated.

Keywords: thyme, quendel, history, multi-compound drug, antimicrobial activity, antimycotic, antioxidative, antiinflammatory, repellent, acaricide 


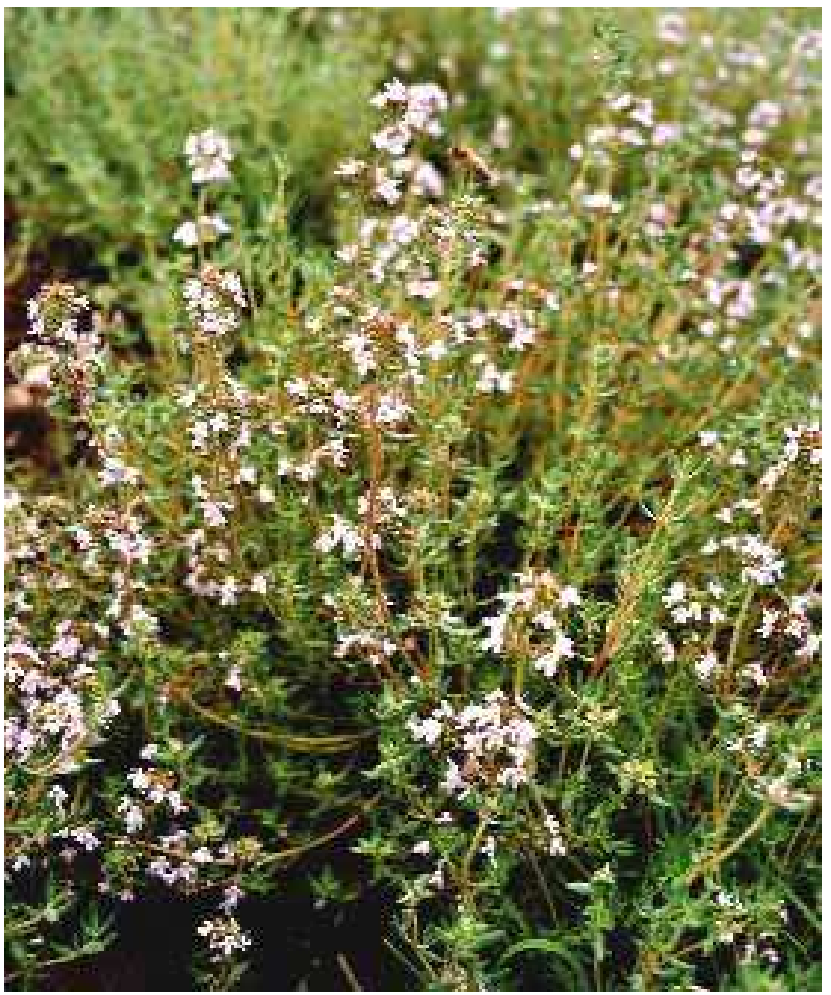

Abb. 1. Der Thymian (Thymus vulgaris) ist ein frostempfindlicher, aromatischer Zwergstrauch der mediterranen Strauchheide.

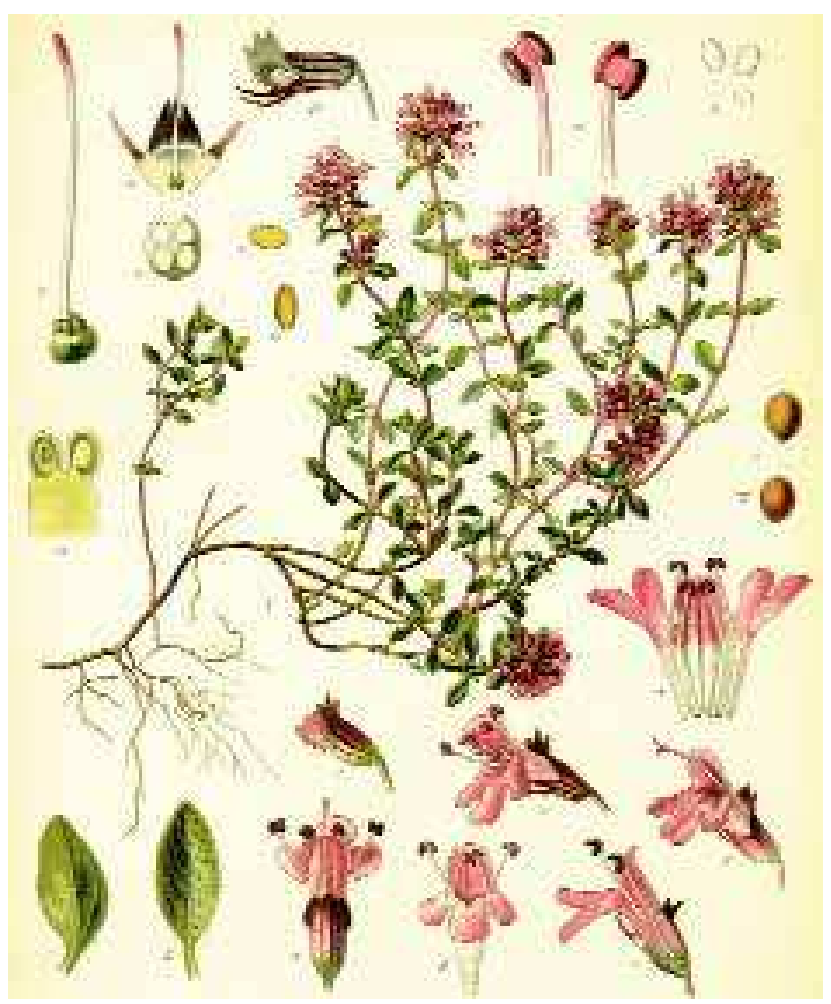

Abb. 2. Der Quendel (Thymus serpyllum), ein naher Verwandter des Echten Thymians, ist nördlich der Alpen heimisch. heide (Macchie). Der Echte Thymian gedeiht im ganzen Mittelmeergebiet bis an die nördliche Grenze der wintermilden Lagen im Kaukasus, die ihm noch eine dauerhafte Besiedlung ermöglichen. Besonders gut gedeiht er auf lockeren, kalkhaltigen Böden. In unseren Breiten friert er in der Regel im Winter ab und muss im Frühjahr von neuem gesät oder gesetzt werden.

\section{Historisches}

Die frühe Geschichte des Thymians verliert sich im Dunkel der Vergangenheit. Weder die Herkunft seines Namens noch die ersten schriftlichen Erwähnungen geben zweifelsfrei Auskunft über die Anfänge seiner Geschichte. In der Antike soll der Thymian oft mit anderen Pflanzen verwechselt worden sein, und für die Herkunft seines Namens wurde neben dem viel zitierten griechischen "thymos" (Mut) oder "thymiama” (Räucherwerk) auch das ägyptischen Zeichen "tham" oder "th" vorgeschlagen. Die erste Erwähnung des Thymians als Heilpflanze fin- det sich bei Dioskurides. Den Römern der Antike war er unter dem Namen Thymus wohlbekannt. Die Ärzte und Köche der römischen Antike schätzten ihn sehr und verwendeten ihn vielseitig. Unter anderem streuten sie getrockneten Thymian unter die Getreidevorräte, um diese mit Hilfe der antiseptischen Eigenschaft des Thymians vor Fäulnis zu schützen und länger haltbar zu machen. In der Medizin setzten sie Thymian vor allem bei Husten, zur Wundheilungsförderung als Magen- und Darmmittel, als Mundwasser und als Wurmmittel ein (Lähmung der Wurmmuskulatur).

In den Kapitularien Karls des Grossen (748-814) bereits aufgeführt, erlangte der Thymian nördlich der Alpen ausserhalb der Klostermauern erst im 12. und 13. Jahrhundert Bekanntheit. In Deutschland wahrscheinlich nicht vor dem 16. Jahrhundert. Bevor der Thymian nördlich der Alpen Einzug hielt, besetzte der Quendel (Thymus serpyllum; nach neuer Nomenklatur $T$. pulegioides), ein naher Verwandter des Thymians, in etwa eben jene Stellung als Arzneimittel, die ihm der Thymian später streitig machen sollte (Abb. 2). In Leonhart Fuchs' „New Kreuterbuch“ von 1543 wird „Thym“ zwar als deutsche Übersetzung des lateinischen und griechischen Thymus erwähnt, das Kapitel trägt jedoch die Überschrift „Von welschem Quendel“ (auch römischer Quendel). Den unzähligen Namen zufolge, die der Quendel (T. serpyllum) im Volksmund des deutschen Sprachraums hatte, war er eine altbekannte und weit verbreitete Arzneipflanze. Häufig beziehen sich die Volksnamen auf die damalige Verwendung. Bezeichnungen wie: „Marikenbettstroh“, „Seelchen der Mutter“ oder „mother of thyme“ weisen den Quendel als eigentliches Frauenkraut aus. Auch Leonhart Fuchs, der alles andere als ein unkritischer Plagiator seiner Zeit war, schreibt im „New Kreuterbuch“ (1543) zum Welschen Quendel: «Er treibt aus die Würm / das Bürdlin / bringt den Frauen ihre Zeit / und zerteilt das gerunnen Blut». In der Veterinärmedizin wird der Quendel (T. serpyllum) zum Teil heute noch als Geburtshelfer eingesetzt. Mit der Verbreitung des Echten Thymians nördlich der Alpen 


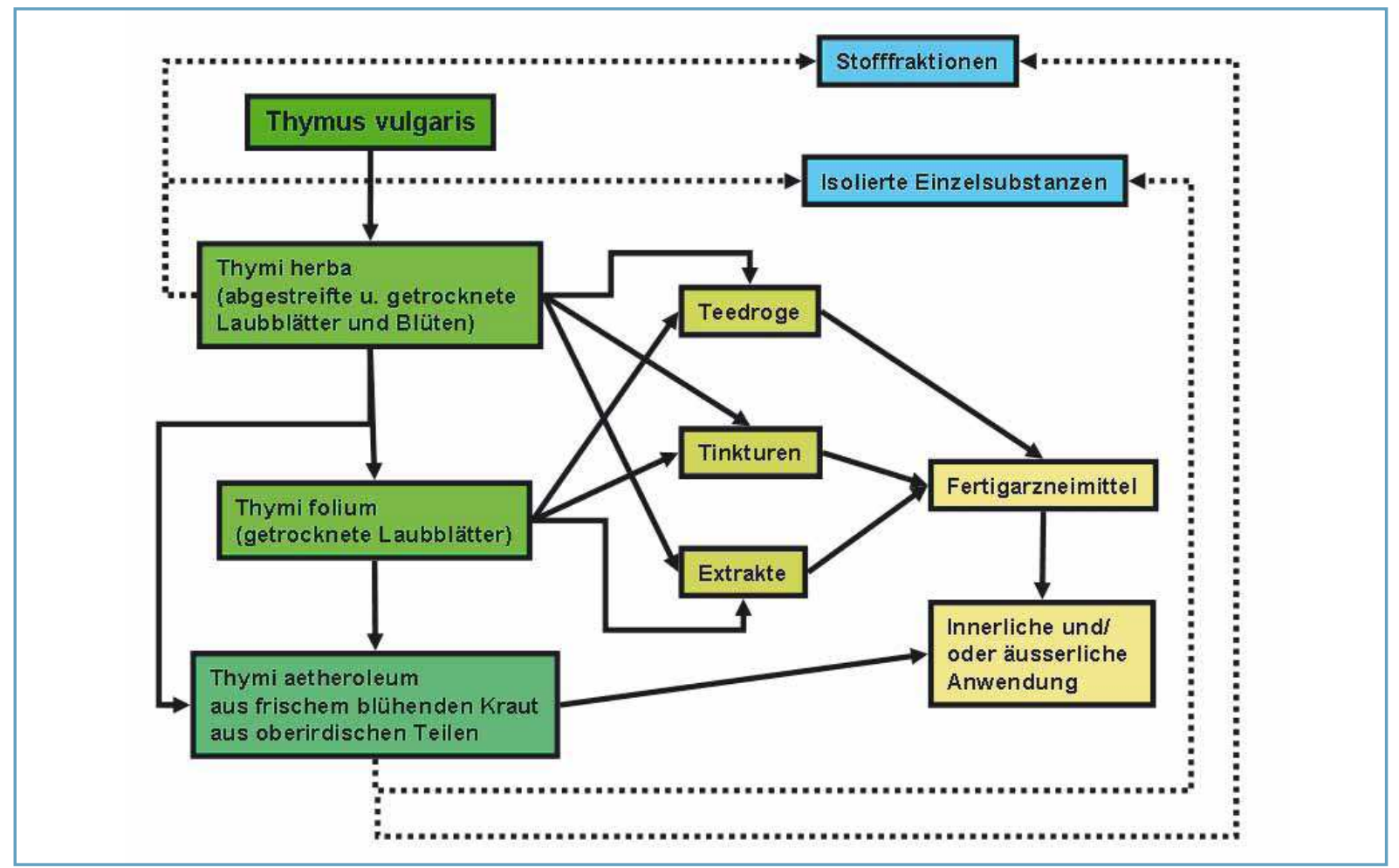

Abb. 3. Thymus vulgaris als Ausgangamaterial für verschiedene Arzneimittelformen, sowie Stofffraktionen und isolierte Monosubstanzen.

geriet der Quendel jedoch in den Schatten seines pharmakologisch etwas kräftigeren Vetters aus dem Süden. Für moderne, phytopharmazeutische Arzneimittel, die Thymian enthalten, werden ausschliesslich Thymus vulgaris und Thymus zygis (Spanischer Thymian) verwendet. Das getrocknete Kraut, das aus gerebelten Blättern oder Blättern und Blüten besteht, findet einerseits als Gewürz Verwendung, andererseits als Droge für Aufgüsse (Tee), Umschläge, Wickel und Bäder oder es dient als Ausgangsmaterial für die weitere Verarbeitung zu Extrakten, Tinkturen oder ätherischem Öl, wobei moderne Fertigarzneimittel überwiegend aus Frischpflanzen hergestellt werden (Abb.3).

\section{Indikationen}

\section{Gestern und Heute}

Die Liste der volksheilkundlichen Anwendungen des Thymians ist lang. Sie reicht weit über die von der ESCOP monographierten Indikationen hinaus (Katarrh der oberen Luftwege, Bron- chialkatarrh, unterstützende Behandlung bei Pertussis sowie Stomatitis und Halitosis). Eine Auswahl der älteren volksheilkundlichen Indikationen und Wirkungen sind etwa: Asthma, Husten, harntreibend, menstruationsfördernd, die Frucht abtreibend, gegen Melancholie, gegen Seitenstechen, bei Fehlsichtigkeit, gegen nervösen Magen, bei Geschwüren und anderem mehr. Je weiter wir in der Geschichte zurückgehen, umso umfangreicher die Liste. Der Umgang mit Krankheiten und Arzneipflanzen war bis in die Neuzeit z.T. auch von magischen und religiösen Interpretationen und von Aberglauben geprägt. Arzneipflanzen waren u.a. fester Bestandteil des religiösen Weltbildes und hatten neben ihrem Erfahrungswert stets auch vielfältige religiös-symbolische und mythologische Bedeutungen. So berichtet eine Volkssage, dass das kräftige Aroma des Quendels böse Geister und sogar den leibhaftigen Teufel vertreibe. An mittelalterlichen Turnieren warf die Dame dem Ritter ihres Herzens ein Sträusschen Thymian zu, das ihm Mut und Kraft verleihen sollte. Ein Teil dieser
Traditionen, Mythen und Legenden, die sich um die Heil- und Schutzwirkung der Pflanzen rankte, fand Eingang in die Kräuterbücher der Antike und des Mittelalters. Erst in der Renaissance begannen einzelne Autoren das bis dahin weitgehend unkritisch übernommene Heilpflanzenwissen der antiken Autoritäten zu hinterfragen und mit eigenen Erfahrungen zu vergleichen, z.B. Leonhard Fuchs in der ersten Hälfte des 16. Jahrhunderts. Einer überbordenden religiösen bzw. abergläubischen Symbolik von Arzneiund Heilpflanzen stehen wir heute äusserst kritisch gegenüber. Wenn wir aber, ungeachtet aller religiösen Verklärungen und im Aberglauben wurzelnden Anwendungen, traditionelle Anwendungen finden, die heute noch Gültigkeit haben (etwa die Anwendung von Thymian bei Bronchitis und Katarrh der oberen Atemwege), mag dies für uns ein Hinweis dafür sein, dass die entsprechende Pflanze bzw. deren Drogen ein wissenschaftlich erfassbares Wirkprinzip enthält. 


\section{Monosubstanzen versus Vielstoffgemische}

Bei vielen Arzneipflanzen mit evidenter Wirkung tut sich die derzeitige, auf Monosubstanzen ausgerichtete Wissenschaft mit der Aufdeckung solcher Wirkprinzipien jedoch schwer. Denn bei sehr vielen Arzneidrogen lässt sich kein einzelner Inhaltsstoff isolieren, dem die Wirkung eindeutig zugeschrieben werden kann. Bei solchen Arzneipflanzen beruht die Wirkung auf dem Zusammenspiel mindestens mehrerer, meist sogar vieler Inhaltsstoffe, die zumeist in sehr niedrigen, mitunter quasi subpharmakologischen Konzentrationen vorhanden sind. Würde man diese Inhaltsstoffe in den entsprechenden niedrigen Konzentrationen isoliert als Monosubstanzen untersuchen, so wären kaum pharmakologische Wirkungen zu erwarten. Zudem scheinen eine Reihe solcher Substanzen bei isolierten Untersuchungen selbst in höheren Konzentrationen als in der jeweiligen Droge bzw. deren Zubereitung keine potentiell therapiespezifischen Wirkungen zu besitzen. Erst im Ensemble und Kontext eines Vielstoffgemisches entwickeln sie ihren spezifischen pharmakologischen und therapeutischen Wert. In der Regel ist der Wirkstoff pflanzlicher Arznei- und Heilmittel ein Vielstoffgemisch mit all den strukturellen und funktionellen Interaktionen der einzelnen Bestandteile des Gemisches. Entsprechend dieser Komplexität der Vielfalt der Bestandteile (Inhaltsstoffe) eines phytopharmakologischen bzw. phytotherapeutischen Wirkstoffes können Phytotherapeutika auch ein breiteres Wirkungsspektrum als Monosubstanzen oder einfache Kombinationen von Monosubstanzen besitzen. Wissenschaftlich nachvollziehbar lassen sich Phytotherapeutika demensprechend als genuine multitarget drugs charakterisieren. Daher muss es nicht überraschen, dass damit auch eine gewisse Vielfalt von Indikationen einhergehen kann.

Arzneipflanzen und daraus hergestellte Arzneimittel (Phytopharmaka) sind stets Vielstoffgemische mit bis zu Hunderten von unterschiedlichen Inhaltsstoffen. Die Inhaltsstoffe lassen sich unter pharmakologischen Gesichts-

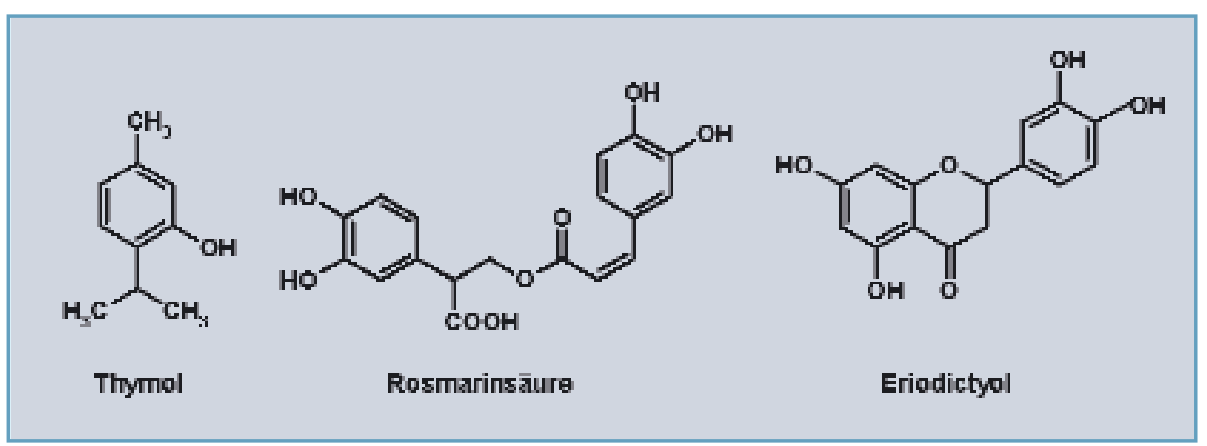

Abb. 3. Dargestellt sind drei Vertreter der pharmakologisch wichtigsten Inhaltsstoffe des Thymians Thymol ist die wichtigste Komponente des ätherischen Öls. Die Rosmarinsäure ist eine in Labiaten weit verbreitete Gerbsäure und das Eriodictyol ist ein Flavanon und gehört zur Gruppe der Flavonoide.

punkten grob in drei Gruppen einteilen: in Wirkstoffe, in bioaktive Substanzen mit potentiell modulierender Wirkung (Begleitstoffe) und in inerte Substanzen ohne eigene Wirkung und ohne pharmakologisch bedeutsame Interaktionen (Ballaststoffe). Auch bei Arzneipflanzen mit bekannten Wirkstoffen können Komponenten, die an sich keine pharmakologisch bedeutsame Wirkung haben, modulierende Effekte ausüben. Die Wirksamkeit und Verträglichkeit kann durch solche Begleitstoffe grundsätzlich sowohl verbessert als auch vermindert werden. In der modernen klinischen Forschung werden derzeit hauptsächlich Kombinationspräparate untersucht, die ein breiteres Wirkspektrum besitzen als Monopräparate und bei denen die Wirksamkeit nicht selten durch synergistische Effekte verstärkt wird (z.B. Thymianextrakte und Primelwurzeln). Beispiele für Kombinationspräparate auf Thymianbasis wären etwa die verschiedenen Bronchipret ${ }^{\circledR}$ Präparate von Bionorica, die zusätzlich Primelwurzel (Primula veris) oder Efeublatt (Hedera helix) enthalten, deren Saponine bei Bronchitis mit übermässiger Verschleimung helfen, den zähen Schleim zu verflüssigen.

\section{Die Wirkstoffe des Thymians}

Als pharmakologisch bedeutsamste Wirkstoffe des Thymians gelten Inhaltsstoffe des ätherischen Öls. Hauptbestandteil des ätherischen Öls sind Thymol (30-70\%) und Carvacrol (3-
$30 \%$, zwei phenolische Monoterpene. Thymol wirkt stark antiseptisch, sekretolytisch, sekretomotorisch und broncholytisch. Thymian ist aber nicht gleich Thymian. Es gibt verschiedene so genannte Chemotypen, die nach ihrer jeweiligen Hauptkomponente benannt werden. Folgende Chemotypen sind bekannt: Thymol-Typ, Carvacrol-Typ, Thuyenol-Typ, Alpha-Terpineol-Typ, Linalool-Typ und Geraniol-Typ. Neben der Namen gebenden Hauptkomponente enthält der Thymian noch eine ganze Reihe weiterer Terpene, darunter p-Cymen und 1,8-Cineol, die die antibakterielle Wirkung des Thymols und Carvacrols synergistisch unterstützen [1]. Weitere Inhaltsstoffe mit pharmakologischer Bedeutung sind die Flavonoide und die Rosmarinsäure (Abb. 3). Flavonoide mit polyphenolischer Struktur, wie das Eriodictyol beispielsweise, fungieren als Radikalfänger (Antioxidans). Die Rosmarinsäure, ebenfalls eine polyphenolische Verbindung, spielt als Antioxidans und Inhibitor der Hyaluronidase bei entzündlichen Prozessen eine wichtige Rolle. Die Vielzahl der ubiquitär vorkommenden Inhaltsstoffe, wie sie auch in anderen Pflanzen vertreten sind, soll hier keine weitere Erwähnung finden.

\section{Pharmakologische Eigenschaften}

\section{Antimikrobielle Aktivität}

Bislang konzentrierte sich die Forschung rund um den Thymian vor allem auf die antimikrobiellen und antioxidativen Eigenschaften. Dabei wurden sowohl 
die Monosubstanzen Thymol und Carvacrol untersucht als auch das ätherische Öl und Extrakte, bei denen es sich um Vielstoffgemische handelt. Die Studien zeigen, dass Thymol, Carvacrol und das ätherische Öl eine hohe antibakterielle Wirksamkeit und ein breites Wirkspektrum besitzen. Die stärkste antibiotische Wirkung entfaltet Thymianöl gegen grampositive Bakterien (z.B.: Staphylococcus aureus und St. epidermidis) und bei einer Reihe pathogener Pilze (z.B.: Candida), sowie gegen einige gramnegative Bakterien wie Klebsiella pneumoniae und Escherichia coli. Die Wirksamkeit-bestimmenden Inhaltsstoffe sind das Thymol und das Carvacrol, zwei phenolische Monoterpene. Die in vitro gemessene antibakterielle Wirkung gegen Erreger wie Moraxella catarrhalis, Klebsiella pneumoniae und Diplococcus pneumoniae mag zumindest teilweise die Wirkung der traditionellen Anwendung bei Bronchitis und Katarrh erklären. Thymol wirkt auch gegen eine Vielzahl von Streptokokken der Mundflora, die für schlechten Atem verantwortlich sind (besonders Streptokokkus mutans). Viele Mundwasser und Zahncremes enthalten Thymol. Ein wässriger Auszug (1:1) des Krautes vermag dosisabhängig Helicobacter pylori zu hemmen [2]. Thymianöl wirkt in vitro noch in einer Verdünnung von 1:2000 gegen Askariden (Spulwürmer). Thymol hemmt das Wachstum Mykotoxin-bildender Pilze der Gattung Aspergillus und Fusarium [3, 4] sowie verschiedener Serotypen der Salmonellen. Diese Erkenntnisse haben das Interesse der Lebensmitteltechnologen geweckt, die nun den Thymian und seine Inhaltsstoffe als potentielles natürliches Konservierungsmittel untersuchen.

Thymianöl ist kein genereller Ersatz für eine notwendige Antibiotikatherapie. In gewissen Fällen können Thymianöl oder Thymianextrakte dennoch eine sinnvolle Alternative darstellen. Nämlich überall dort, wo der Einsatz von Antibiotka unverhältnismässig ist, wo eine Keimreduktion beispielsweise ausreicht, etwa bei der Prophylaxe gegen Infektionen kleiner Verletzungen, bei Entzündungen in Mund und Rachenbereich oder bei einer einsetzenden Bronchitis. Denn der leichtfertige und unsachgerechte Umgang mit Antibiotika hat in den letzten Jahrzehnten dazu geführt, dass sich resistente Keime ausbreiteten, und einige wichtige Antibiotika bereits ihre Wirksamkeit verloren haben. Mit Hilfe von Thymianöl bzw. Thymianextrakt als Prophylaxe lässt sich oftmals eine Infektion abwenden - ohne gravierende Nebenwirkungen und ohne das Risiko, resistente Keime zu selektieren. Dass eine solche Anwendung mitunter nicht ausreicht, spricht nicht grundsätzlich gegen sie. Das unangebrachte Hinauszögern einer (notwendigen) Antibiotikatherapie ist allerdings ebenso $\mathrm{zu}$ vermeiden wie ihr leichtfertiger Einsatz. Eine weitere Möglichkeit, die antibiotische Wirkung $\mathrm{zu}$ erhöhen und gleichzeitig die $\mathrm{Ne}$ benwirkungen sowie das Risiko der Selektion resistenter Keime zu verringern, stellen Kombinationen ätherischer Öle mit Antibiotika dar. GIORDANI et al. gelang es auf diese Weise, die antifungale Wirkung von Amphotericin B mit Hilfe von Thymianöl erheblich zu steigern [5]. Einzelne Bakterienstämme vermögen sich in Gegenwart subletaler Dosen isolierter Komponenten des Thymianöls zumindest bis zu einem gewissen Grad zu adaptieren, was in Anbetracht des äusserst plastischen Stoffwechsels der Bakterien kaum überrascht. So änderte Bacillus cereus in Gegenwart subletaler Dosen von Carvacrol die Zusammensetzung der bakteriellen Membranlipide in der Weise, dass die durch Carvacrol verursachte Erhöhung der Membranfluidität wieder weitgehend aufgehoben war [6]. Inwieweit eine solche Adaptation für eine allfällige erworbene sekundäre Resistenz verantwortlich sein könnte, bleibt vorerst dahingestellt. Mit Resistenz- und Rückstandsproblemen zu kämpfen, haben auch die Imker. Ein Teil der Bienenvölker ist bereits von Akarizid-resistenten Varroa-Milben befallen. Die Suche nach Alternativen und der damit zusammenhängenden Beforschung ätherischer Öle hat zu zwei in der Schweiz registrierten Produkten auf Thymianbasis geführt: "Api Life VAR", ein Kombinationspräparat aus Thymol, Eucalyptol, Kampfer und Menthol, sowie "Thymovar" mit dem Wirkstoff Thymol [7].

\section{Eigenschaften als Repellents}

Thymianöl zeigt bei topischer Anwendung bereits bei einer Konzentration von nur $0.05 \%$ einen etwa $90 \%$ igen Schutz gegen die Stechmücke Culex pipiens pallens [8]. Fünf Monoterpene des Thymians wurden genauer auf ihre insektenvertreibende Eigenschaft (Repellents) hin untersucht. AlphaTerpinen und Carvacrol haben sich dabei gegen die Stechmüche Culex pipiens pallens als wirksamer erwiesen als das kommerzielle Vergleichsprodukt Deet [9]. Ein Nachteil des Thymianöls ist seine relativ kurze Wirkdauer im Vergleich zu Deet, das bis zu 8 Stunden wirksam sein soll.

\section{Antiphlogistische Wirkung}

Die Indikation Wundheilung sucht man im Kapitel Thymus vulgaris in den meisten Kräuterbüchern vergeblich. Neuere tierexperimentelle Untersuchungen weisen jedoch auf wundheilungsfördernde Eigenschaften des Thymians hin. Dursun et al. untersuchten die wundheilende Wirkung von Thymianöl an Ratten und stellten fest, dass Thymianöl NO-induzierte überschiessende Entzündungsprozesse hemmt und die Wundheilung beschleunigt [10]. Thymol hemmt zudem die COX-1- und COX-2-vermittelte Prostaglandinsynthese [11]. Des Weiteren wird durch die Rosmarinsäure dosisabhängig die Hyaluronidase gehemmt, die bei entzündlichen Prozessen die Permeabilität des Gewebes erhöht [12].

\section{Antioxidative Wirkung}

Thymianöl und Thymol besitzen, wie In-vitro- und In-vivo-Studien zeigen, ein ausserordentlich hohes antioxidatives Potential. In einem In-vitro-Modell schnitt Thymol etwa vergleichbar gut ab wie alpha-Tocopherol [13]. Im Tierversuch schützte Thymianöl die mehrfach ungesättigten Fettsäuren der Zellmembran aller untersuchten Organe sehr effektiv vor Oxidation und wirkte dadurch Alterungsprozessen entgegen [14]. Die antioxidativen Eigenschaften des Thymianöls und des Thymols haben die Nahrungsmittelindustrie, die auf der Suche nach natürlichen Antioxidantien ist, dazu veranlasst, Thymianöl und Thymol als potentielles natürliches Antioxidans zu beforschen [15]. 


\section{Spasmolytische, sekretolytische und tonisierende Wirkung}

Neuere Ex-vivo-Untersuchungen an der Meerschweinchentrachea bestätigen die spasmolytische Wirkung wässriger und alkoholischer Thymianextrakte $[16,17]$. Thymianöl fördert dosisabhängig die Sekretion seröser Interziliarflüssigkeit und steigert die Ziliartätigkeit in den Bronchien. Zur tonisierenden Wirkung des Thymianöls, die vor allem in der Aromatherapie von Bedeutung ist, liegt eine aktuelle tierexperimentelle Untersuchung von LIM et al. vor. Sie konnten zeigen, dass inhaliertes Thymianöl bei Mäusen eine signifikante Steigerung der motorischen Aktivität bewirkt [18]. Diese zentralnervöse Wirkung ist wahrscheinlich an die Wahrnehmung des Geruchs gebunden, denn anderweitige Experimente mit Mäusen, deren Geruchssinn chemisch vorübergehend ausgeschaltet wurde, zeigten auf Duftexpositionen keinerlei Verhaltensänderungen [19]. Beim wässrigen Aufguss (Tee) spielen neben dem schwer wasserlöslichen und deshalb eher untervertretenen ätherischen Öl vor allem die Flavonoide (Thymonin, Cirsilineol und 8-MethoxyCirsilineol) sowie die Bitter- und Gerbstoffe die entscheidende Rolle. Sie sind wahrscheinlich auch für einen Teil der gastrointestinalen Wirkung, die die Droge als Tee entfaltet verantwortlich (Stomachikum und Karminativum).

\section{Toxikologie}

Obwohl phenolische Verbindungen wie Thymol und Carvacrol für ihr toxisches Potenzial bekannt sind, liegen kaum zuverlässige Berichte über gravierende Nebenwirkungen des ätherischen Öls vor. Eine Erklärung für die gute Verträglichkeit des Thymianöls haben möglicherweise SASAKI et al. gefunden. Sie konnten zeigen, dass die beiden potentiell toxischen Verbindungen Thymol und Carvacrol bei Mäusen eine Steigerung der Aktivität der beiden Phase-II-Enzyme Glutathion-STransferase (GST) und Quinon-Reduktase (QR) induzieren [20]. Diese PhaseII-Enzyme dürften massgeblich an der Detoxifizierung xenobiotischer Verbindungen beteiligt sein und besitzen wahrscheinlich auch antikanzerogene Eigenschaften. Akute toxische Reak-

Tab. 1. Dosierungsangaben zur Anwendung von Thymian

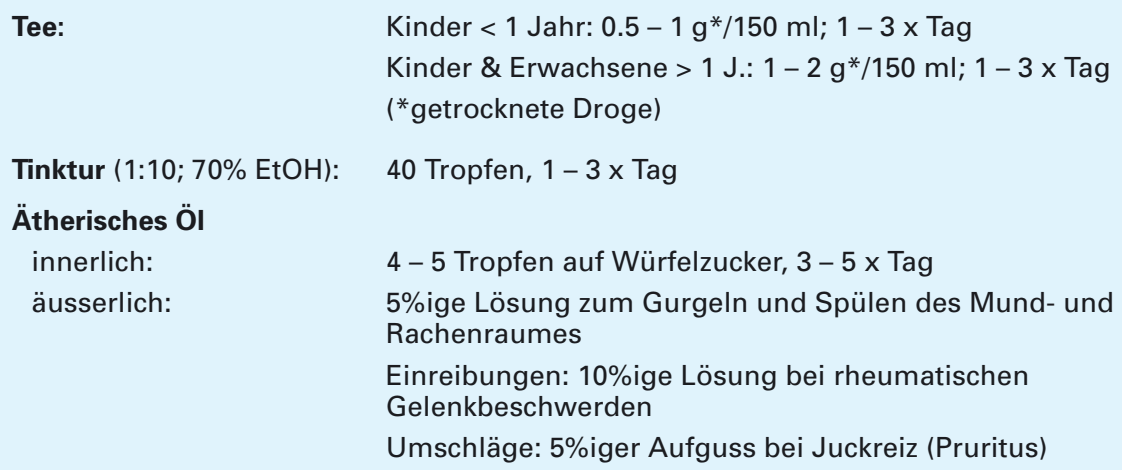

tionen bei äusserlicher Anwendung des ätherischen Öls in Form von Hautirritationen und allergischen Reaktionen sind möglich, aber offensichtlich selten. Reines Thymol soll bei der innerlichen Anwendung höherer Dosen zu Bauchschmerzen und kollapsartigen Zuständen führen. Die ESCOP (European Scientific Cooperative on Phytotherapy) rät Schwangeren und Stillenden von der innerlichen Anwendung vorsichtshalber ab. Nach bisherigen Erkenntnissen führt die Einnahme von 20 Tropfen Thymianöl jedoch zu keinen akuten oder chronischen toxischen Effekten. Bei Kindern bis zum Alter von drei Jahren sollte die Anwendung ätherischer Öle bei topischer Anwendung im mund- und nasennahen Bereich dennoch vermieden werden, da ätherische Öle reflektorisch einen Laryngospasmus auslösen können. Unbedenklich dagegen ist die Zubereitung als wässriger Aufguss (Tee) sowohl für Kinder wie für Schwangere. Die LD50-Dosis bei oraler Gabe von Thymol liegt bei Ratten bei $0.98 \mathrm{~g} / \mathrm{kg} \mathrm{KG}$, für Thymianöl bei $2.84 \mathrm{~g} / \mathrm{kg} \mathrm{KG}$.

\section{Allgemeines zur Anwendung}

Ätherische Öle stellen eine hochkonzentrierte Form der flüchtigen Pflanzeninhaltsstoffe dar. In dieser Form sind sie gut haltbar, für eine unmittelbare Anwendung aber nur beschränkt geeignet. Von einer inneren Anwendung hoher Dosen ätherischen Öls über längere Zeit muss grundsätzlich abgeraten werden, da Thymol ein geringes neurotoxisches Potential besitzt. Für die innerliche Anwendung als Stomachikum oder Karminativum eignen sich besonders wässrige Auszüge des Krautes (Tee) und Tinkturen (Tab. 1). Die ESCOP empfiehlt, bis zu dreimal täglich 40 Tropfen der Tinktur mit etwas Wasser einzunehmen. Steht nur ätherisches Öl zur Verfügung, können vier bis fünf Tropfen auf einen $\mathrm{Zu}$ cker geträufelt mit etwas Wasser eingenommen werden. Zur Herstellung einer Lösung, die zum Spülen oder Gurgeln von entzündetem Mund- und Rachenraum verwendet werden soll, sind Tinkturen wegen der alkoholvermittelten besseren Löslichkeit geeigneter als ätherische Öle. Aus $5 \mathrm{~g}$ Thymian und $100 \mathrm{ml}$ heissem Wasser lässt sich aber ebenfalls eine wirksame Gurgellösung herstellen. Bei der äusserlichen Anwendung des Thymian-Öls gilt es, die haut- und schleimhautreizenden Eigenschaften zu beachten. ThymianÖle sollten deshalb mit einem Trägeröl, z.B. Mandelöl, auf 3-5\% verdünnt werden, bevor sie aufgetragen werden. Bei der äusserlichen Anwendung stehen die antimikrobiellen, die hyperämisierenden und wundheilungsfördernden Eigenschaften im Vordergrund.

\section{Fazit}

Die Verwendung des Thymians bei Bronchitis und Katarrh der oberen Luftwege, die sich wie ein roter Faden durch alle Epochen der traditionellen Volksmedizin zieht, konnte durch experimentelle Forschung bestätigt wer- 
den. Leider mangelt es zurzeit noch an klinischen Studien, die Auskunft über die medizinische Relevanz geben [21]. Wie die jüngste Forschung zum Thymian zeigt, ist mit den traditionellen Indikationen das Potential des Vielstoffgemisches Thymian und seiner unterschiedlichen Arzneiformen noch lange nicht ausgeschöpft. Besonders zur antibiotischen Wirkung, zur Wundheilung und als potentielles Konservierungsmittel (antibakterielle und antioxidative Eigenschaften) liegen viel versprechende Ergebnisse aus der experimentellen Forschung vor. Auf weitere Ergebnisse aus der noch jungen Thymianforschung darf man gespannt sein.

Der vorliegende Artikel entstand im Rahmen eines von der "Stiftung für Komplementärmedizin" unterstützten Projektes zu Thymus vulgaris.

\section{Literatur}

1. Pina-Vaz C, Goncalves Rodrigues A, Pinto E, Costa-de-Oliveira S, Tavares C, Salqueiro L, et al. Antifungal activity of Thymus oils and their major compounds. J Eur Acad Dermatol Venereol 2004;18(1):73-8.

2. Tabak M, Armon R, Potasman I, Neeman I. In vitro inhibition of Helicobacter pylori by extracts of thyme. J Appl Bacteriol 1996 80(6):667-72.

3. Soliman KM, Badeaa RI. Effect of oil extracted from some medicinal plants on different mycotoxigenic fungi. Food Chem Toxicol 2002;40(11):1669-75.
4. Nguefack J, Leth V, Amvam Zollo PH, Mathur SB Evaluation of five essential oils from aromatic plants of Cameroon for controlling food spoilage and mycotoxin producing fungi. Int $\mathrm{J}$ Food Microbiol 2004;94(3):329-34

5. Giordani R, Regli P, Kaloustian J, Mikail C, Abou L, Portugal H. Antifungal effect of various essential oils against Candida albicans. Potentiation of antifungal action of amphotericin B by essential oil from Thymus vulgaris. Phytother Res 2004;18(12):990-5.

6. Ultee A, Kets EP, Alberda M, Hoekstra FA, Smid EJ. Adaptation of the food-borne pathogen Bacillus cereus to carvacrol. Arch Microbiol 2000;174(4):233-8.

7. Imdorf A, Blumer-Meyere P. Lässt sich Varroa destructor mit ätherischen Ölen bekämpfen? In: Schweizerisches Zentrum für Bienenforschung, Liebefeld (3003 Bern); 2006.

8. Choi WS, Park BS, Ku SK, Lee SE. Repellent activities of essential oils and monoterpenes against Culex pipiens pallens. J Am Mosq Control Assoc 2002:18(4):348-51.

9. Park BS, Choi WS, Kim JH, Kim KH, Lee SE. Monoterpenes from thyme (Thymus vulgaris) as potential mosquito repellents. J Am Mosq Control Assoc 2005;21(1):80-3.

10. Dursun N, Liman N, Ozyazgan I, Gunes I, Saraymen $\mathrm{R}$. Role of Thymus oil in burn wound healing. J Burn Care Rehabil 2003; 24(6): 395-9.

11. Marsik P, Kokoska L, Landa P, Nepovim A, Soudek $\mathrm{P}$, Vanek T. In vitro inhibitory effects of thymol and quinones of Nigella sativa seeds on cyclooxygenase-1- and -2-catalyzed prostaglandin E2 biosyntheses. Planta Med 2005;71(8):739-42.

12. Ippoushi K. Evaluation of Inhibitory Effects of Vegetables and Herbs on Hyaluronidase and Identification of Rosmarinic Acid as a Hyaluronidase Inhibitor in Lemon Balm (Melissa officinalis L.). Food Sci. Technol. Res. 2000;6(1):74-77.

13. Lee KG, Shibamoto T. Determination of antioxidant potential of volatile extracts isolated from various herbs and spices. J Agric Food Chem 2002;50(17):4947-52.
14. Youdim KA, Deans SG. Effect of thyme oil and thymol dietary supplementation on the antioxidant status and fatty acid composition of the ageing rat brain. Br J Nutr 2000; 83(1):87-93.

15. Burt S. Essential oils: their antibacterial properties and potential applications in foods - a review. Int J Food Microbiol 2004:94(3): 223-53.

16. Boskabady $\mathrm{MH}$, Aslani MR, Kiani S. Relaxant effect of Thymus vulgaris on guinea-pig tracheal chains and its possible mechanism(s). Phytother Res 2006;20(1):28-33.

17. Meister A, Bernhardt G, Christoffel V, Buschauer A. Antispasmodic activity of Thymus vulgaris extract on the isolated guinea-pig trachea: discrimination between drug and ethanol effects. Planta Med 1999:65(6) 512-6.

18. Lim WC Seo JM, Lee Cl, Pyo HB Lee BC Stimulative and sedative effects of essentia oils upon inhalation in mice. Arch Pharm Res 2005:28(7):770-4

19. Tsuchiya T, Tanida M, Uenoyama S, Nakayama Y, Ozawa T. Effects of olfactory stimulation on the sleep time induced by pentobarbital administration in mice. Brain Res Bul 1991; 26(3):397-401

20. Sasaki K, Wada K, Tanaka $Y$, Yoshimura $T$ Matuoka K, Anno T. Thyme (Thymus vulgaris L.) leaves and its constituents increase the activities of xenobiotic-metabolizing enzymes in mouse liver. J Med Food 2005:8(2):184-9.

21. Ernst E, Marz RW, Sieder C. [Acute bronchitis: effectiveness of Sinupret. Comparative study with common expectorants in 3,187 patients]. Fortschr Med 1997;115(11):52-3.

\section{Korrespondenzadresse:}

Felix Iten, dipl. Biologe

UniversitätsSpital Zürich

Dep. für Innere Medizin

Institut für Naturheilkunde

Rämistrasse 100, CH-8091 Zürich

felix.iten@usz.ch 